\title{
Software development of Rubber recipe management database
}

\author{
Hongna $\mathrm{LI}^{1, \mathrm{a}}$, Huan $\mathrm{LIU}^{2, \mathrm{~b}}$ \\ ${ }^{1}$ Maritime College, Tianjin University of Technology, Tianjin 300384, China \\ ${ }^{2}$ Maritime College, Tianjin University of Technology, Tianjin 300384, China \\ aemail: lihongna2005@126.com, bemail:2289970487@qq.com
}

\begin{abstract}
Keywords: Recipe management of Rubber production; database of InterBase; Delphi Software Development
\end{abstract}

\begin{abstract}
Discuss how to manage the production of rubber formula, realize the Rapidity and efficiency the production formulation. Based on InterBase database and Delphi software development, connecting with the actual operation, introduce in detail the steps in the process of database management software development, provide a guiding framework of how to develop a practical database management software. Finally through an actual management of the new formula, verify the feasibility and the convenience of this software. For the factory the actual drug weighing plays an important role.
\end{abstract}

\section{Introduction}

This software in order to realize changing and setting up recipe database rapidly, at the same time, should consider the combination of the hardware. Although more involved in the software database about the operations, but the same good to match other hardware information. When reading should consider the combination of the hardware. Although more involved in the software database about the operations, but the same good to match other hardware information. When reading informations between hardware and software, which control software is based on combination of another database and PLC, from already set database information and the corresponding PLC logic instruction, the corresponding drive for the raw material storage cabinet of the factory, so as to achieve the aim of rubber raw materials weighing drug matching[1]. Because of the characteristic of the InterBase combined with the Delphi closely, so can easily build the development environment of InterBase database. Delphi VCL control provides a convenient and rapid development process, with the visual interface, improve the development efficiency and shorten the development cycle greatly.

\section{The overall structure of the recipe management system}

The design of the Rubber recipe formulation management system, mainly by four main modules, that is divided into Raw materials and formulation module, System information management module, Production development and Query module, SQL language access database module. As shown in figure 1.

\section{- Raw materials and formulation module(include Materials Table and Recipe Table)}

This module is the core of the software, Its function is to develop a production formula, modify the raw material, so that to read database on rubber drug weight control software, thus weighing the drug. Here mainly involves weighing order、 drug quality of weighing drug errors that allowed、 mixing places and a series of weighing information set[2].

When the production, or production of rubber raw material changes, such as : raw discontinued、raw material replacement, require manual changes to the database software tabular information. Remove unused raw materials, use of new materials information input: mainly to join the material type, material number, material name and other information. 


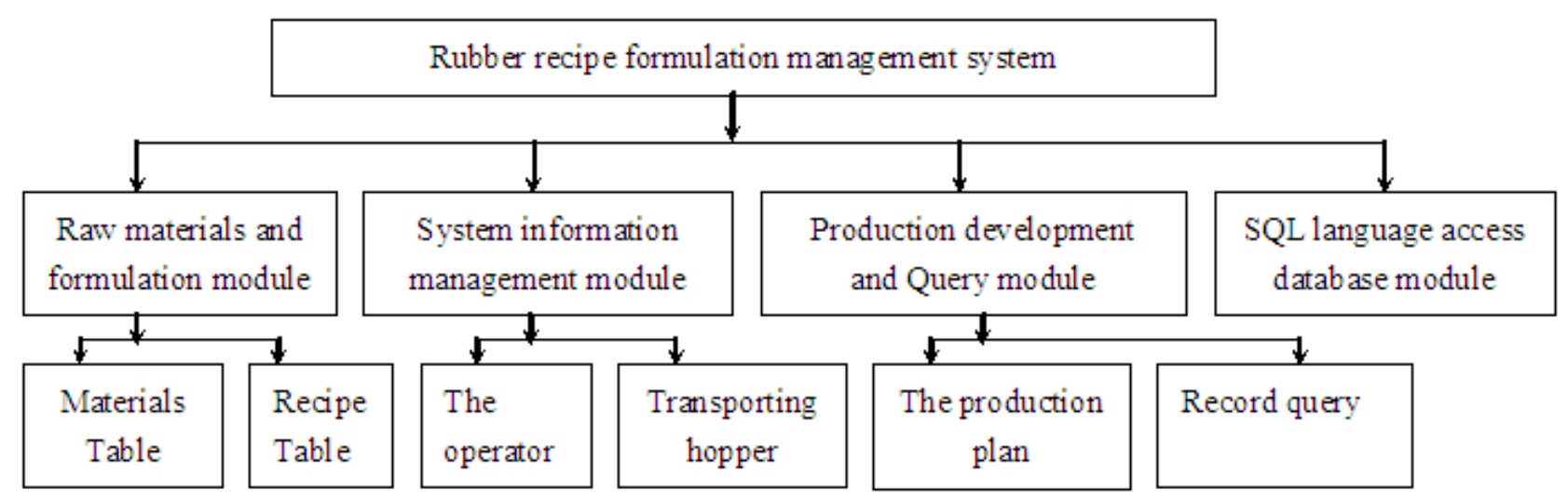

Fig.1. overall structure of the recipe management system

\section{- System information management module(include The operator and Transporting hopper)}

This module mainly manages the drug weight operator's account password information. To set up the drug's position in the storage cabinet, because of the different drugs ranks number in the loading position is different, so the location of the drug is like the information of the database software, such information to read and drive can match.

- Production development and Query module (include The production plan and Record query)

Through this module, you can set the time specified, specify the recipe, specify the number of production plans, and query the historical information that has been completed. This facilitates the setting of production plan, and improves the efficiency of historical data query.

By using the software, Monitor not only can make the production plan on duty that day, but also can develop other time production plan, and set the production quantity for each recipe, can also be cancelled to remove the production plan.

\section{- SQL language access database module}

In the input box ,you can write the corresponding SQL statement, so as to realize the data display software does not implement and supplement the deficiency of software design.

\section{Design flow formulation management system and key technology}

The management system runs under the Windows 7, takes the InterBase database as the background, uses the Delphi software as the system development tool, Because of the high efficiency of Delphi + InterBase software, the software development cycle is greatly reduced.

\section{- Design flow of system}

According to the different rubber production technology, rubber models required for different drugs and different quality, in the formulation of the interface, according to the required product information, we should set up the material formulation information, the design process mainly includes the following steps of production[3]:

A: Design the structure of material database table. Displays the table information in the specified form. At the same time for all the displayed information is also required to obtain, such as material name, material number, to display data in other forms in the call.

B: Design database hopper table structure. Mainly reflected the correspondence between the hopper number and drug raw materials. When adding new drugs, new drugs need to be modified in the storage cabinet, and the 60 hoppers and 60 raw materials are modified, and the one-to-one corresponding.

C: Design the structure of formula database table. When the production of different types of rubber, according to the formula number, system can screened information such as formula names 、weight 、 allowing the error 、 weighing sequence of all the materials needed.

\section{- Key technology of formulation management system}

Key technology is mainly the connection problem of database files and Delphi and the combination of database SQL language and programming language[4]. Generate the corresponding 
FastReport report file and print it out through the database display, to browse or save the display information. Report function is primarily achieved through the FastReport VCL, and combined with simple code language, this is another important reason why software development efficiently.

A: Database technology

For the InterBase database, it can be used across the platform, and the installation is fast, and most of the database languages are general and general database[5]. This is the advantage of it. However, it also has certain database data types, for example: this software mainly uses the date (Date), time (Time) and time stamp (Timestamp) and other data types, these data types are different from the present database language, so it is important to select the database when the database is based on the time. For different tables in the database, the structure and design is based on the actual production need, add and modify data one by one. The structure of this software is mainly related to the table data structure as shown in Figure 2.

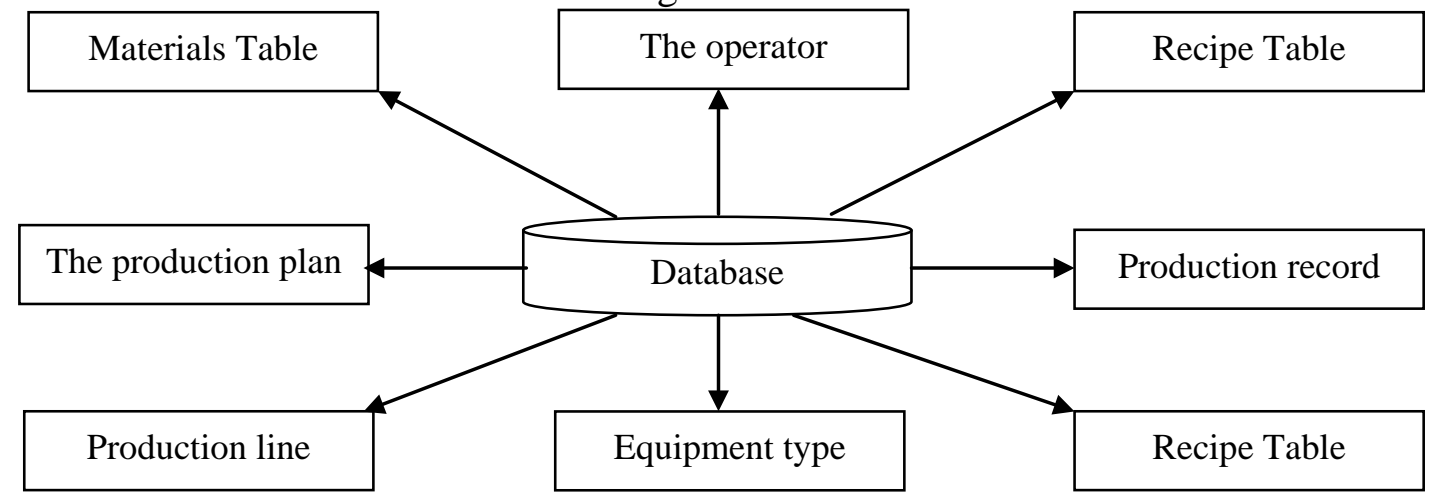

Fig.2. Illumination of database structure

B: Developing software technology

Delphi compared with other programming software, it can be called the shortest development time-consuming type of software. Rich VCL, especially formulated a InterBase VCL, its interface is very easy to set InterBase database, the code is simple and clear. Use most of the code, as well as software code to access the database information, as follows:

\begin{tabular}{|l|l|l|}
\hline $\begin{array}{c}\text { Controls the } \\
\text { name }\end{array}$ & \multicolumn{1}{|c|}{ attribute } & \multicolumn{1}{c|}{ value } \\
\hline \multirow{4}{*}{ TIBDatabase } & DataBaseName & The database path \\
\cline { 2 - 3 } & DefaulTransaction & IBTransaction \\
\cline { 2 - 3 } & Params & $\begin{array}{l}\text { user_name=account } \\
\text { password= password }\end{array}$ \\
\cline { 2 - 3 } & LoginPrompt & False \\
\cline { 2 - 3 } & Connected & True \\
\hline TIBTransaction & Double click & Read Committed \\
\hline \multirow{4}{*}{ TIBTable } & Database & IBDatabasel \\
\cline { 2 - 3 } & TableName & $\begin{array}{l}\text { Choose to display } \\
\text { the table }\end{array}$ \\
\cline { 2 - 3 } & Active & True \\
\hline DataSource & DataSet & IBTable \\
\hline DBNavigator & DataSource & DataSource \\
\hline DBGrid & DataSource & DataSource \\
\hline
\end{tabular}

Tab.1. software code to access the database information

\section{System interface}

For the entire software development, mainly involves the basic form of Delphi design control, based on the attribute information of the corresponding control configuration, can form the content of the show. In order to reflect concise and simple principle, as far as possible simplify the 
information content displays, the combination of menu bar and toolbar improves the speed of using software for drug management, and some form is shown in Figure 3.

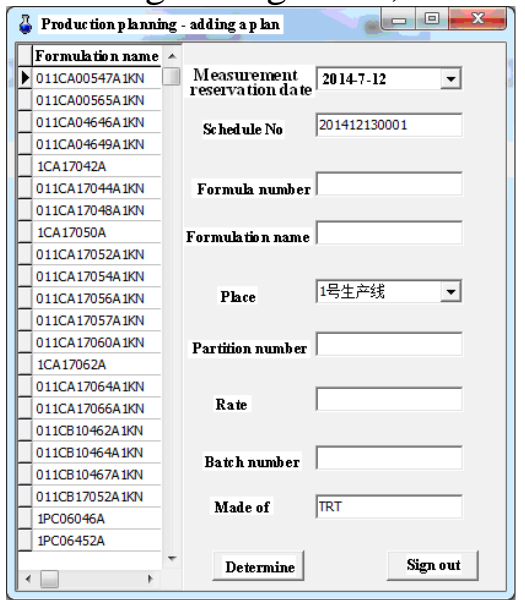

(a) Production plan adding interface

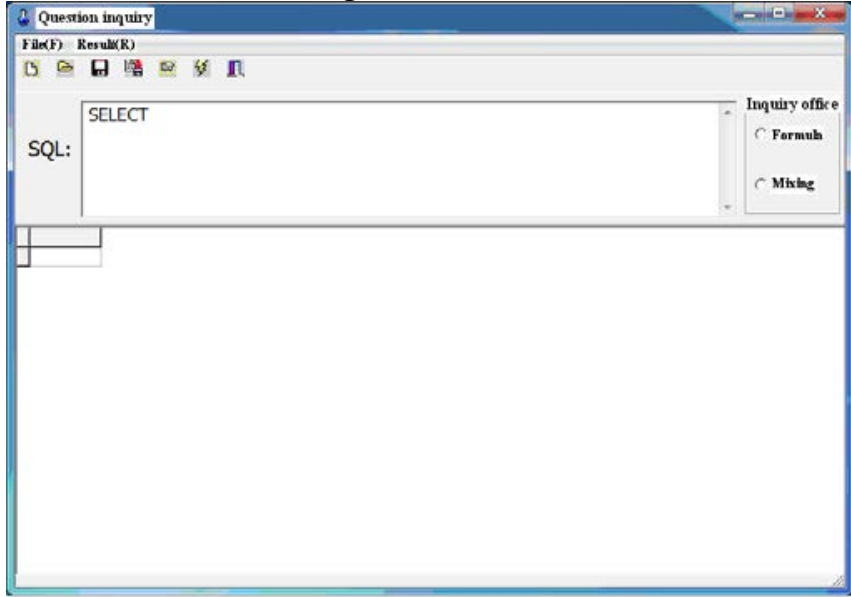

(b) SQL statement access database interface

Fig. 3 Some forms showing of system

\section{Conclusion}

This article discusses the different functions of different modules of database software, as well as explaining the software structure of the database and software design steps and connection method Delphi and InterBase database file. This software mainly realizes the revision of raw material information, as well as the formulation of the production formula, production plan and so on, so as to save the data in the database file, and then according to the designated production plan for production and processing.

\section{Acknowledgement}

In this paper, the research was sponsored by the 2012 Colleges and Universities in Tianjin Science and Technology Development Fund Program (Project No. 20120801) and Teaching Reform Project of Tianjin University of Technology (Project No. YB13-35).

\section{References}

[1] Jan.Jib.Image Processing, Recongstruction, restoration concept sand met [M]. BocaRaton, FL:

Taylor\&Francis,2006.11.

[2] Bigun,Josef.Vision with dierction:A Systematic Introduction to Image Processing and com[M].Berin Springer:2006.9.

[3] XIE Hong,WANG Hongqiang.Development of Control Software for NC Operating Platform Design Based on Visual Basic and Delphi.[J].Machine and electron,2002(2).

[4]Wood ,JohnW.Multidimensional Signal,Image,AvdVideo Processing and Coding[M].Boston Elsevier Academic Presscessing .2006.5.

[5]HUANG Sui ,LIU Jian.Design and Implementation of an InterBase and Delphi-based Dentistical X-ray Image Proceessing System[J].Computer Engineering,2002.28-6. 